\title{
Ni Una Menos, más allá de la marcha, el campo en disputa’
}

Recibido: $20 / 08 / 2019$

Aprobado: 19/10/2019
FANNI MUÑOZ CABREJO

Pontifia Universidad Católica del Perú

famunoz@pucp.edu.pe

\section{RESUMEN}

El presente artículo se propone estudiar el movimiento Ni Una Menos Perú 2016, con el objetivo de identificar y analizar el juego de tensiones y negociaciones que se presentan entre las diversas colectivas feministas que participaron en la marcha del 13 de agosto del 2016. En esta se evidencian distintas maneras de hacer política entre las colectivas y las organizaciones feministas institucionalizadas, en un contexto globalizado, del surgimiento de los movimientos sociales en red, y en una sociedad autoritaria como la peruana. Asimismo, en esta interacción se observa cómo el género se intersecta con la raza, clase y la etnicidad. Intento plantear algunas hipótesis que expliquen cómo este movimiento, que como indica Melucci (1994, p. 121), se inscribe en los nuevos movimientos sociales, y se distancia de formas tradicionales de organización política. Así, Ni Una Menos se transformó en un escaparate donde miles de mujeres se atrevieron a narrar situaciones de violencia en las que fueron sometidas, y donde participaron diversas colectivas y organizaciones feministas, que finalmente terminaron fragmentándose. Este estudio es una primera aproximación desde una perspectiva cualitativa.

Palabras clave: Perú, género, feminismos, movimientos sociales, movimientos en red, colectivas feministas, violencia de género, raza, clase y etnicidad.

\section{Not one less, beyond the march, the field of dispute}

\begin{abstract}
The article sets out to study the Ni Una Menos Perú 2016 movement with the objectives of identifying and analyzing the game of tensions and negotiations between the several feminist collectives that participated in the August 13, 2016 march. This march presents different ways of carrying out politics between new collectives and established feminist organizations in a context of global relations, social networks, and within the authoritarian Peruvian society. This interaction also reveal the intersection between gender and race, class, and ethnicity. The study seeks to address how a movement that became a platform for thousands of women who brave to narrate violence accounts to which they were subjects, become fragmented. The study consists of seven in-depth interviews to members of the most active and diverse collectives.
\end{abstract}

Keywords: Peru, gender, feminisms, social movements, online movements, feminist collectives, gender-based violence, race, class, and ethnicity.

* Esta ponencia fue presentada en International Symposium: Comparative Perspectives on \#MeToo University of Kentucky, Lexington, KY, USA. April 8-9, 2019. Agradezco a Brenda Reyna, Vanessa Laura y Angélica Motta por su colaboración en este estudio. 


\section{Surgimiento de Ni Una Menos (NUM)}

H 14 de julio del 2016, el vídeo donde Arlette Contreras es arrastrada de los cabellos por Adriano Pozo no fue prueba suficiente para que la Corte Superior de Justicia de Ayacucho dictamine una pena de acuerdo con grado de violencia ejercida; por el contrario, fue sentenciado a solo un año de prisión suspendida. Al siguiente día, 15 de julio del 2016, la Primera Sala para Reos en Cárcel de la Corte Superior de Justicia de Lima condenó a Rony García a cuatro años de prisión suspendida por haber agredido de forma brutal a Lady Guillén (cuyo verdadero nombre es Lizeth Rosario Socia Guillén) en el 2012.

Ambos actos de impunidad del sistema de justicia peruano frente a hechos evidentes de violencia hacia las mujeres provocaron la indignación colectiva de todo el país, la cual devino en la marcha NUM, la más multitudinaria que se haya realizado en el Perú en defensa de los derechos de las mujeres. Según cálculos de los medios de comunicación, unas 150 mil personas en Lima y provincias salieron a las calles ese 13 de agosto.

NUM en el Perú se explica por varias razones. Algunas han sido señaladas en los testimonios que hemos recogido de feministas provenientes de distintos espacios. De acuerdo con el testimonio de las activistas feministas vinculadas a la política partidaria, señalan que una de ellas es la presencia continua de movilizaciones en el contexto nacional y regional que en el periodo 2015 y 2016, en respuesta a las medidas laborales que el Estado pretendió imponer y al intento de Keiko Fujimori de salir elegida presidenta de la república. Dicho periodo se caracterizó por la presencia continua de movilizaciones, tanto en el contexto nacional como el regional. En el Perú podemos citar las marchas contra Ley Pulpín ${ }^{1}$ (Ley N. ${ }^{\circ}$ 303288), en el 2015, y Keiko No Va, en el 2016, lideradas por jóvenes universitarios con filiación política de izquierda, pero que obtuvieron la adhesión mayoritaria de la población. Si bien en ambas marchas se hizo uso de las redes sociales para la convocatoria y la organización, sus líderes mantuvieron el modelo de toma de decisión de las asambleas, con las que lograron consolidar sus victorias: la derogación de la Ley Pulpín

1 La Ley N. ${ }^{\circ} 30288$, Ley que Promueve el Acceso de Jóvenes al Mercado Laboral y a la Protección Social, conocida como la Ley de Régimen Laboral Juvenil o Ley Pulpín, fue presentada en el 2015. Su objetivo era que los jóvenes de dieciocho a veinticinco años ingresen al mercado laboral formal, pero sin beneficios laborales como el reconocimiento de gratificaciones, compensación por tiempo de servicios (CTS) y vacaciones. Tras varias movilizaciones, el 26 de enero del 2015 el Congreso derogó la ley. 
y la derrota de la candidata Keiko Fujimori en las elecciones presidenciales del 2016.

Si bien estas movilizaciones no tenían carácter feminista, fueron espacios en los que distintas colectivas feministas convergieron, y como resultado de ello se creó la plataforma de organizaciones y el grupo de Facebook "Mujeres Resistencia” (entrevista SDLC, 27 de febrero del 2019). Asimismo, en los últimos años se vienen impulsando colectivas feministas, así como la expansión del movimiento LGBT y la teoría queer que favorecieron el surgimiento de NUM.

Por otro lado, en la última década en los países de la región, Europa y los Estados Unidos existe un horizonte de movilizaciones en defensa de la violencia contra las mujeres, como Ni Una Menos de Argentina (2015) y México (2016), y el \#Metoo (2018). Argentina es el primer país donde se realiza la primera marcha NUM, como consecuencia de los feminicidios de María Eugenia Lancetti y Chiara Páez. El NUM argentino, para Natalucci y Rey (2018, p. 4), es una manifestación, a la vez una consigna y un colectivo de mujeres "donde se articulan [...] organizaciones profesionalizadas, activistas y mujeres sin pertenencia orgánica ni trayectorias previas que se incorporaron al ciclo".

No obstante, el detonante del surgimiento de NUM en el Perú fue el sentimiento de indignación que originó entre la población nacional la imagen ya señalada de Arlette Contreras arrastrada de los cabellos por Adriano Pozo. Esto provocó la respuesta de feministas y activistas feministas pertenecientes a colectivas que convergieron en la movilización.

M, feminista y filósofa egresada de la Pontificia Universidad Católica del Perú (PUCP), y proveniente de la clase media alta limeña, publica un post en su cuenta de Facebook que expresa y canaliza el sentimiento de indignación frente a la imagen, la situación de violencia y la impunidad por parte del Poder Judicial, en el que convoca y llama a la acción: “¿Cuándo tomamos la calle, hermanas?, ¿cuándo decimos nosotras «ni una menos»?” (M, 2016; en Caballero, 2018, p. 103)

Este llamado en Facebook se viralizó y a los pocos días más personas y grupos, tanto en las redes virtuales como fuera de ellas, se sumaron a la convocatoria de movilización, en respuesta a la incapacidad del Estado para sancionar la violencia como delito. A la par, distintos actores comenzaron a autoorganizarse, dando lugar a expresiones de reciprocidad, intercambio y colaboración entre las y los participantes que se plasmó en la entrega libre del logo de la marcha, donaciones de merchandising, avisos gratuitos en paneles 
publicitarios, spots, performances, actividades para generar recursos, organización de especialistas para atender a testimonios, publicación de adhesiones a la marcha, etc.

De esta forma, la marcha NUM dejó de ser una iniciativa de un grupo para convertirse en una inmensa red de voces heterogéneas, corales y multiconectadas vía smartphones. Supo así aglutinar movimientos tradicionales, partidos, sindicatos, colectivos, grupos religiosos, redes informales de amigos, individuos sueltos, medios de comunicación, entre otros, bajo un mismo objetivo común: acabar con la violencia de género hacia las mujeres.

Al no estar convocada por una organización específica, difícilmente se puede pensar la marcha NUM como un actor feminista homogéneo. Por el contrario, las mujeres que impulsaron inicialmente la convocatoria en redes sociales eran feministas y activistas feministas, por lo que "[la marcha] no significó el desarrollo de una identidad feminista generalizada, sino más bien una identidad con muchas voces, diversos repertorios y agendas políticas", según Natalucci Rey (2018, p. 17), coincidiendo con el NUM argentino.

Así, el feminismo que acompañó a NUM estuvo conformado por una diversidad de mujeres muy jóvenes, activistas independientes o en colectivos en Lima y provincias, que conscientes de su opresión luchan contra el sistema de dominación masculino desde sus espacios, las calles y las redes, combinándolas con el ciberactivismo.

El feminismo institucionalizado, asociado a las ONG feministas, no fue protagonista de esta movilización, pero cabe destacar que la demanda principal de esta: la lucha contra la violencia de género, ha sido uno de los temas prioritarios de la agenda feminista nacional desde sus orígenes hace más de treinta años y que había conseguido avances en el Estado a través de la dación de normas e implementación de políticas públicas.

En el caso de NUM Perú, se consiguió transformar la indignación ciudadana frente a la violencia contra las mujeres en empoderamiento gracias a los testimonios publicados en el grupo cerrado Ni Una Menos Movilización Nacional Ya, en el Facebook. Este espacio fue creado inicialmente para coordinar la marcha, pero se convirtió en una vitrina donde miles de mujeres se atrevieron a narrar las situaciones de violencia de las que fueron protagonistas, en su gran mayoría agresiones sexuales producidas en su infancia por familiares cercanos. Los testimonios originaron una catarsis colectiva y demostraron que en nuestra sociedad la violencia de género no es un hecho individual sino un problema estructural. 
Las historias de violencia fueron el catalizador que llevó a las mujeres a pasar de la indignación a la necesidad de actuar y de empoderamiento, es decir, a la capacidad de reconocer violencia en su vida cotidiana, rechazarla y ayudar a otras a defenderse de ella. Por algo, el hashtag inicial \#NiUnaMenos incluyó luego las expresiones \#VivasNosQueremos y \#TocanAUnaTocanATodas.

Tanto Gutiérrez (2016) como Caballero (2018) coinciden en señalar que no se trata de una movilización social. Mientras para Caballero se trata de una acción colectiva, basándose en Tarrow, para Gutiérrez (2016, p. 38) la marcha evidenció el cambio del paradigma político y social que atraviesa América Latina donde las tecnologías digitales han adquirido un papel destacado al configurar un nuevo prototipo de participación, creación e imaginación política que opera sobre causas específicas con nuevos actores que se mueven en espacios híbridos. Asimismo, NUM no entraría en la categoría movilización social, de acuerdo con Caballero.

No obstante, para Gutiérrez (2016, p. 7) “[...], existe un problema para conceptualizar los nuevos movimientos sociales, por ello, uno de los conceptos más apropiados para definirlos sería el de 'movimiento red', acuñado por Arnau Monterde, que hace referencia a los nuevos ecosistemas sociales basados en 'nuevas formas de cooperación' y no en 'grandes dogmas ideológicos"”.

NUM, de acuerdo con la conceptualización de Castells, y en la cual se sustenta este artículo, sería un movimiento social en red, que fue uno de los principales medios de canalización de la protesta. Castells (2004, p. 91) identifica los movimientos sociales de la sociedad red, los cuales tienen que ser analizados "por lo que dicen ser". Es decir, por las prácticas discursivas de los individuos y los procesos en los que se hallan inmersos y están definidos por los "actores sociales que aspiran a un cambio cultural (cambio de valores)" (Castells, 2009, p. 394) y de creencias que implica una transformación en las mentalidades y que afectan al conjunto de la sociedad. Son movimientos emocionales que se van a formar "mediante un proceso de comunicación autónoma, libre del control del poder institucional" (Castells, 2012, p. 27). Y donde las redes sociales digitales "ofrecen la posibilidad de deliberar y coordinar acciones sin trabas" (Castells, 2012, p. 27), y se van a relacionar con la sociedad.

A esta autonomía también se mostró un rechazo a ser definido como un colectivo u organización, que no tenía voceras oficiales, o coordinadoras nacionales e internacionales, y que no pretendía ser una voz autorizada de las 
miles de mujeres que sufren violencia todos los días. No obstante, esta respuesta generó dos frentes al interior de la movilización: uno que se adaptó a la propuesta y otro que la interpretó como una contradicción.

En la contradicción, de acuerdo con los testimonios que se han recogido para este estudio, se señala una relación vertical entre las impulsoras de NUM y el conjunto de activistas y colectivas feministas que no formaban parte de grupo del núcleo duro de la marcha. Dicha verticalidad quedó expuesta en las asambleas y denunciada en los espacios de coordinación virtual (chats de Facebook o Messenger, así como fan page y grupos de Facebook) durante y después de la marcha; y que alcanzó su pico más alto al identificar el intento de registro del nombre NUM ante Indecopi por parte de la feminista J, que participó en el grupo impulsor inicial.

Desde que se iniciaron las críticas, las integrantes del grupo impulsor, según algunas de ellas señalan, optaron por no responder y mantuvieron una actitud dialogante, insistiendo en que NUM es una plataforma que sirve para visibilizar la violencia que viven las mujeres y exigir al Estado cumplir su deber de erradicarla, basada en la autoorganización y autoconvocatoria, no en liderazgos individuales, bajo la misma dinámica con la que se organizaron las marchas NUM en Argentina, Chile y México. Tras el incidente de Indecopi, muchas de las activistas y colectivas participantes abandonaron NUM y continuaron con sus actividades en sus respectivos grupos y colectivas.

\section{Campo de tensión entre diversas colectivas feministas}

El movimiento NUM también se tiene que comprender en el marco del surgimiento de los movimientos feministas en las últimas décadas, tanto en la región como en Europa y Estados Unidos, como el \#MeToo, y los movimientos NUM que se han creado en México y Argentina. En todos ellos llama la atención su masividad. En el caso peruano se pueden identificar aproximadamente más de ochenta "colectivas feministas", como se denominan, que han ido surgiendo en la última década. Cabe destacar que como consecuencia de NUM se constituyeron nuevas colectivas y grupos NUM en las zonas de Lima Norte, en barrios populares como San Juan de Lurigancho, Comas, Los Olivos, entre otros.

Al igual que lo que señala Luna Follegatti para el caso de Chile (2018), se tiene que hacer una nueva lectura del movimiento feminista que dé cuenta de su auge a partir del siglo XXI. Las nuevas "colectivas feministas" plantean una 
ruptura generacional con relación a las llamadas feministas institucionalizadas. Mientras estas se encuentran vinculadas al Estado y su lucha es por la desigualdad de las mujeres y por el seguimiento y vigilancia de los derechos sexuales y reproductivos, así como las políticas de violencia de género en un proceso de democratización por parte del Estado, las nuevas colectivas irrumpen en la escena con diversos discursos vinculados a las representaciones que las mujeres tienen sobre sí mismas, asociadas a la libertad del control de sus cuerpos, a la importancia de lo comunitario barrial, a la diversidad sexual, al cuidado, a la lucha contra la violencia (especialmente la violencia letal como es la feminicida), a la transformación de la mujer como sujeto político, y a la performatividad como expresión corporal. Así se desmarcan del Estado. No obstante, existen algunas líneas de continuidad que se inscriben en el discurso contra la violencia de género y los derechos sexuales y reproductivos. Maruja Barrig (2019) denomina a estas colectivas feministas como feministas contraculturales.

Como bien ha señalado Nancy Fraser en una reciente entrevista en Madrid (España), se vive "un momento especial, una nueva ola en la formación del feminismo con enorme potencial de cambio respecto de las dos décadas anteriores". Para Maruja Barrig (2019) estas colectivas feministas se inscriben en un feminismo contracultural.

Si bien la historia del movimiento feminista en la región se ha caracterizado por momentos de mayor activación y otros de silenciamiento, incluso en su ocaso en la década de 1990; no obstante, el proceso que se viene produciendo en los últimos años, evidencia otro contexto y da cuenta de la configuración de diversas colectivas que, si bien tiene un núcleo en la participación estudiantil, también lo conforman otros grupos vinculados a sectores populares. Se trata de colectivas diferenciadas o dispersas, historia que también forma parte del movimiento feminista en el Perú, pero a diferencia de la caracterización que hizo Virginia Vargas (2008) identificando tres vertientes en el feminismo peruano: i) La vertiente feminista, ii) La vertiente popular y iii) la vertiente política, en la actualidad se podría pensar en una nueva forma de agruparlas como i) feministas institucionalizadas del siglo XX y las ii) feministas del siglo XXI, en donde hay una heterogeneidad de grupos.

Las colectivas están formadas por personas que proceden de los amplios y diversos sectores de las nuevas clases medias que han surgido en el Perú en el contexto del crecimiento económico, así como también convergen otros de sectores populares. La edad de las mujeres que participan en estos colectivos comprende entre los diecinueve y cuarenta años, ubicándose también mujeres 
mayores. Se trata de mujeres que cuentan o siguen estudios universitarios o superior técnica, así como han finalizado la secundaria completa. Son grupos pequeños que se forman con la finalidad de hacer cosas muy concretas. No obstante, de acuerdo con los testimonios, estas colectivas se han constituido a partir de espacios más grandes en los que venían participando, como el caso de Déjala Decidir (2014). Otras se desarrollan en espacios universitarios y, en los últimos años, provienen de zonas barriales, como es el caso de algunas colectivas: las Insurgentes y las Poco Floro, cuyas integrantes son de Lima Norte o Lima Sur.

Asumen diferentes formas de ser feministas y el feminismo tiene un significado para cada generación que da cuenta de la pluralidad de los feminismos. En esta definición es que se afirma su pertenencia a determinada clase social, sus identidades étnicas, raciales y sexuales, que se manifiesta en el lenguaje que emplean. Así, por ejemplo, para una feminista de treinta años, mujer, que se asume como una "híbrida" porque puede transitar entre el mundo de las clases medias altas y las pertenecientes a sectores populares, de padres profesionales migrantes, ella transita entre un distrito popular y otro de sectores medios, y concibe su feminismo de la siguiente manera:

Para mí ser feminista es bacanazo. Para mí, ser feminista implica que yo no solo quiero el feminismo para fuera de la casa y para la calle, sino que también lo quiero en mi vida personal, en mi cama, en mis alimentos, en cómo miro la vida. Es súper para mí ser feminista y entender las desigualdades entre mujeres y que se busca erradicar el sistema patriarcal. Es un proyecto de vida (entrevista a U, 1 de marzo del 2019).

Mientras que para otra joven de procedencia popular, el feminismo estaría asociado a una experiencia de vida sin necesidad de pasar por la academia:

Yo creo que tenía cierta vergüenza ante la idea de que me asociaran a las feministas de entonces. Era como que... Tenía la idea de que las feministas eran esas mujeres institucionalizadas, que eran de la universidad, y que no podían ser de otra manera, que estaban muy enfrascadas en los libros, generando grandes textos para reflexionar. No pensaba que eso fuera..., ese tipo de feminismo no me identificaba. Pero no conocía otra cosa del feminismo. Entonces, con el movimiento de Ni Una Menos, empecé a entender que podían generarse plataformas de articulación entre mujeres, que además podían decirse feministas por su experiencia de vida, y que no había un solo feminismo, sino había diferentes formas de ser feministas (entrevista a C, 27 febrero del 2019). 
Y para una activista feminista de cincuenta años, académica, de sector medio alto, que ha transitado en el pasado en el feminismo institucionalizado, y que ahora pertenece a NUM, define que:

El feminismo sigue siendo una posibilidad, ya quizá no tanto a nivel personal, porque más o menos como que ya puedo darme ahora la libertad, y dado que ya existe la transnacionalización, etc., de poder activar en otros grupos, otros grupos de otros países y demás. La esperanza que yo podría tener con el feminismo en el Perú es que por fin despierte el espíritu feminista de las mujeres peruanas (entrevista a C, 27 de febrero del 2019).

Entre las referencias de feministas emblemáticas señalan a Marcela Lagarde, Rita Segato, Virginia Woolf y Björk, esta última por ser activista mediombientalista, creativa y consecuente. En el Perú, a Rocío Muñoz, Virginia Vargas y las mujeres de organizaciones populares.

Una característica que articula a estas "colectivas" es su experiencia de vida en la sociedad en red global, que como señala Castells (2009, pp. 66-67), "es la contraposición de la lógica de la red global y la afirmación de la multiplicidad de identidades locales [...]. Se observa que como tendencia principal es la diversidad histórica y cultural: fragmentación más que convergencia". Se observa que no todas las colectivas cuentan con un espacio físico; más bien se comunican a través del uso del Facebook, Twitter, hacen uso de la calle como experiencia vital para exigir las reivindicaciones, así como de una nueva forma de hacer política que se caracteriza por el asambleísmo.

Entre estos grupos podemos señalar a los siguientes: Mujer Dispara Audiovisual (2016), Awqa Feminista (2017), Collera Red (2017), Fugitivas (2016), Ni Una Menos Carabayllo (2017), Ni Una Menos Los Olivos (2017), Ruray. Colectiva Feminista Barrial en San Martín de Porres (2017), Abolofem Perú, Asamblea de Mujeres y Diversidad Lima, Awqa Feminista Colectiva Comunitaria, Awqa Warmi (2017), Manada Feminista (2015), Colectivo Alfombra Roja (2013), Comando Feminista, (2013), Alfombra Roja (Perú e Italia), Colectivo No tengo Miedo (2014), Asociación de Mujeres Carabayllanas, Colectivo Parió Paula Percusión, Grupo de Mujeres Diversas, La Purita Carne, Las Insurgentes, Las Respondonas (2016), LIFS-Lesbianas Independientes, entre otras (ver Barrientos y Muñoz, 2018, p. 291).

En los nombres escogidos por las colectivas como Manada Feminista o Comando Feminista se evidencia una nueva construcción identitaria, un nuevo empleo del lenguaje. Las estrategias de shock de cuerpos desnudos en las 
puestas en escena de sus protestas y performances es otra de sus características. Hecho que contrasta con "la racionalidad de las feministas del primer milenio que «deben vestirse de funcionarias" si quieren interactuar con el Estado (Barrientos y Muñoz, 2018, p. 291). La idea de trabajar en grupos más pequeños es porque de acuerdo con los testimonios se podría realizar más cosas y tener más impacto en la lucha de la erradicación de la violencia de género, la lesbofobia, la transfobia (entrevista a U, 1 de marzo del 2019).

De acuerdo con los testimonios, la creación de colectivas diversas se explicaría porque algunas personas tienen una necesidad de reconocimiento y el trabajo de hormiguita no les gusta porque se "prefiere ser abeja reina dentro del propio grupo" (entrevista a C, 27 de febrero del 2019). Según esto, la necesidad de reconocimiento por parte de grupos postergados del acceso al poder y la democratización de las redes hacen posible la proliferación de estas colectivas.

Entre estas colectivas se observa una fuerte red de interrelaciones, ya sea virtuales o físicas, con colectivos de otros países de la región o también de Europa, específicamente en España. En el caso de una representante de NUM: C, activista feminista de larga trayectoria, quien mantiene relaciones en Madrid, en Casa de América, con Women's Foreign Policy Network, y con La Caja de Pandora. Otro es el caso de U, entre otras, que tiene contacto con feministas argentinas como Marian Lacono y Marta Dillon.

Y si bien hacen alianzas con las feministas institucionalizadas, la mayoría de integrantes de las colectivas considera que este no es el camino a seguir. De acuerdo con la opinión de una feminista activista que pertenece a NUM, su primer interés de participar en este colectivo fue tender puentes entre el feminismo institucionalizado del siglo XX y los nuevos grupos, pero después de observar que las posiciones son irreconciliables por la incapacidad de dialogar y de entendimiento, "llega a constatar que dicha unión entre el feminismo del siglo XX y del XXI no se llegue a dar" (entrevista a C, 27 de febrero del 2019).

\section{Campo de tensiones y conflictos que se presentan: etnicidad, raza, clase y género}

Como ya se ha señalado previamente, la organización de la marcha y las posteriores acciones de conformación de NUM produjeron una serie de desencuentros al interior del grupo impulsor y las diferentes colectivas que participaron. 
Esta crisis y ruptura evidencia problemas hondos que el país no ha resuelto, como son el racismo, el autoritarismo y la ausencia de prácticas democráticas, que se entrelazan con la historia del feminismo en el Perú, caracterizada por la polarización entre distintos grupos: la división entre burguesas y populares.

Así, en la actualidad, se observa una fragmentación entre las distintas colectivas y NUM. Este último no tiene la capacidad de convocar y articular a las diferentes colectivas porque se ha producido una división. El grupo impulsor que promovió la marcha es denominado por otras colectivas como el "grupo de las blancas". Asimismo, varios de los testimonios de este estudio mencionan que este grupo no hace un activismo feminista, tienen un comportamiento poco abierto al diálogo y, por el contrario, está orientado a una vocación autoritaria.

De acuerdo con la narrativa de los testimonios y la información recogida, el desencuentro entre las distintas colectivas y NUM está asociado con los elementos de etnicidad, raza, clase, que se refleja en el testimonio de una de las entrevistadas:

Yo creo que el problema, que es bastante grave, es la división del grupo de mujeres por cuestiones, por ejemplo, de interseccionalidad de clase y raza. Entonces esa es una gran fractura que creo que está al origen de la primera ruptura que tuvo este germen de Ni Una Menos (entrevista a C).

Asimismo, otros testimonios dan cuenta de la imposibilidad que se tiene de "aceptar las diferencias". No es casual que surjan denominaciones como "las blancas", las feministas "marrones" y las "híbridas". Las que proceden de barrios populares sienten que son rechazadas por las feministas blancas y que ellas son autoritarias, no horizontales y privilegiadas. Esta ruptura se ha llegado a explicitar este año en la marcha convocada por NUM bajo el nombre de Mujeres Libres de Violencia (17 de agosto del 2019), y en la cual las diferentes colectivas no solo no participaron en la marcha, sino que difundieron por redes un pronunciamiento "de deslinde con Ni Una Menos Perú: Tocan a Una, Tocan a Todas". En este documento cuestionaron al llamado "grupo minoritario" por prácticas verticales que no propician la participación "colectiva y popular de obreras, estudiantes, afroperuanas, indígenas, campesinas, lesbianas, trans y todas las mujeres y diversidades que seguimos organizándonos en colectivos, plataformas, sindicatos y asambleas".

Se observa cómo estas diferencias étnicas, de clase y raza, son las que explicarían esta jerarquización que se presenta entre los grupos. Como señala 
Marisol de la Cadena (1991, p. 9), "Las identidades étnicas se construyen en interacciones, de acuerdo con atributos que se reconocen y se fijan, conflictivamente, en la relación".

\section{Algunas reflexiones finales}

- Uno de los logros que se perciben como resultado del movimiento NUM es que se produce una visibilización y difusión pública de la violencia de género hacia las mujeres, tanto en sectores del Estado, la ciudadanía y los medios de comunicación. Este tema se ha posicionado como un tema de agenda central en estos actores y se ha plasmado en políticas específicas para atender la situación de violencia de género hacia las mujeres.

- El crecimiento de las distintas colectivas feministas se ha venido conformando en las últimas décadas, incrementándose en número de colectivas después de la marcha del NUM (13 de agosto del 2016), y los objetivos que persiguen, para mostrar una ruptura con el movimiento feminista del siglo XX. Las nuevas colectivas conformadas por jóvenes, en su gran mayoría urbanas universitarias, sin dejar de mencionar colectivas de sectores populares, que se reagrupan, se renombran, no tienen local, no tienen jerarquías y, en una palabra, transitan. No buscan la institucionalidad, son contrarias al Estado y se interesan por tener seguidores, especialmente, a partir de las redes, irradiando su impacto en la opinión pública.

- En las universidades ha surgido la necesidad de erradicar la violencia de género, impulsadas por diversas colectivas, al igual que lo que viene sucediendo en otros países de la región y donde se vienen presentando denuncias públicas por acoso sexual. Asimismo, se viene observando que las universidades no siempre están preparadas para responder a esto de manera efectiva.

- Entre las características que se identifican en estos grupos, una primera aproximación general que requerirá a futuro un análisis más exhaustivo de cada grupo, especialmente de los que han surgido en los sectores populares, nos permite señalar los aspectos comunes que se observan en ellos y en los cuales se expresan tensiones propias de una sociedad de experiencia de modernidad transitando a una posmoderna, 
y en la que se están presentando nuevas dinámicas de organización y acción social, bajo una economía fragmentada, formal, informal y narcodelictiva.

- A pesar que algunas investigaciones señalan que el NUM no es un proceso que ha nacido al interior del movimiento feminista ni se inscribe en él, este estudio permite inferir que en el Perú este es un movimiento en el cual las feministas y distintas colectivas impulsaron el NUM. Por ello, este movimiento tiene que ser comprendido en el marco de los nuevos feminismos, diferentes a los que se conocía, con mayor complejidad.

- Por otro lado, es importante entender este movimiento del siglo XXI en el marco de lo que señala Castells: movimientos sociales en red. En esta línea, tal como señala Caballero (2018, p. 229): "Facebook ofrece a los usuarios la posibilidad de compartir testimonios, opiniones y experiencias de vida, con un número potencialmente gigantesco de receptores y también permite expresarles su solidaridad". No obstante, las redes pueden llevar a la fragmentación antes que a la convergencia. Las entrevistadas mencionan que las redes generarán espacios de disputa y conflictos, donde la despersonalización contribuye a que estos conflictos se den a niveles muy altos y denigrantes: "La contra de las redes es que puede generar peleas y distancias y disputas políticas en un nivel tan fuerte que resulta paupérrimo. Hay cosas que he leído que me parecen de verdad denigrantes" (entrevista a U). Esto conlleva a que los grupos puedan volverse espacios de violencia y poco amigables para las personas que conviven en ellos.

- NUM no ha tenido un impacto político mayor en la lucha contra la violencia de género hacia las mujeres. Los conflictos que se ha presentado, especialmente los referidos a la ruptura por la etnicidad, clase y raza, no han favorecido que sea la instancia que articule a las distintas colectivas alrededor de un objetivo común. Las mujeres de clases altas como las de sectores populares tuvieron grandes problemas para comunicarse entre ellas; ambos grupos se negaban a tomar como válida la experiencia del otro y se basaban en la desconfianza y el miedo. Asimismo, se encontró en las mujeres de sectores populares una fuerte estigmatización de la mujer "privilegiada", lo que reflejaría problemas estructurales de la sociedad peruana como el racismo y el autoritarismo. Para las mujeres feministas de los sectores populares, la lucha fe- 
minista y de izquierda se corresponde con una identidad de una mujer "chola" o "marrón" de sectores populares o periféricos. Estas características han sido señaladas por la historiadora Cecilia Blondet (1995), cuando hacía referencia a una disputa de clases entre las "feministas políticas" y las "feministas burguesas".

- Asimismo, esta nueva ola del feminismo a la que todavía no se le puede dar un nombre, pero que se caracteriza por su orientación antiEstado, aún no ha encontrado una estrategia que le permita actuar bajo una nueva forma de hacer política con un impacto más efectivo. Los nuevos grupos feministas se encuentran en la paradoja de optar entre la trascendencia de ir consolidándose como movimientos para poder reivindicar sus derechos, o la inmanencia de lo efímero en que surgen sin tener mayor continuidad. 


\section{Bibliografía}

Annunziata, R., Arpini, E., Gold, T. y Zeifer, B. (2016). Argentina. En Sorj, B. y Fausto, S. (Comps.). Activismo politico en tiempos de internet. São Paulo: Edições Plataforma Democrática. Recuperado de http://www.plataformademocratica.org/Arquivos/ Activismo_politico_en_tiempos_de_internet.pdf.

Barrig, M. (2019). Investigaciones sobre empleo y trabajo femenino. El Arriero, entrevista a Maruja Barrig (27 de marzo).

Barrientos, V. y Muñoz, F. (2018) Las nuevas voces de los feminismos: hacia una cartografía del feminismo en el segundo milenio. En Bidaseca, K. (Coord.). Poéticas de los feminismos descoloniales desde el sur (pp. 275-306). Red de Pensamiento Decolonial (RPD).

Blondet, C. (1995). El movimiento de mujeres en el Perú 1960-1990. En Cotler, J. (Coord.). Perú 1964-1994. Economía, sociedad y política. Lima: Instituto de Estudios Peruanos.

Caballero, G. (2018). Redes sociales y feminismos en la acción colectiva: el caso de Ni Una Menos en el Perú [tesis de maestría en Sociología]. Lima: Pontificia Universidad Católica.

Cabral, P. y Acacio, J. (2016). La violencia de género como problema público. Las movilizaciones por Ni Una Menos en la Argentina. Questión. Revista especializada en Periodismo y Comunicación, 1(51), 170-187.

Castells, M. (2004). La era de la información. Economía, sociedad y cultura. Volumen II: El Poder de la Identidad. Ciudad México: Siglo XXI Editores.

Castells, M. (2009). Comunicación y poder. Madrid: Alianza Editorial.

Castells, M. (2012). Redes de indignación y esperanza. Los movimientos sociales en la era de internet. Madrid: Cultura Libre.

De La Cadena, M. (1992). Las mujeres son más indias: etnicidad y género en una comunidad del Cuzco. Revista Isis Internacional, Ediciones de las Mujeres, 16. Santiago de Chile.

Diaz, N. y López, A. (2016). Ni Una Menos: el grito en común [tesina de investigación]. Buenos Aires: Universidad Nacional de la Plata, Facultad de Periodismo y Comunicación Social.

Follegati, L. (2018) El constante aparecer del movimiento feminista. Reflexiones desde la contingencia, Pp. 77-97. En Zerán, F. (Ed.). Mayo feminista. La rebelión contra el patriarcado. Feminismos y género. Santiago de Chile: Ciencias Sociales y Humanas LOM.

Fuller, N. (2004). La relación entre la academia y el feminismo. En Flora Tristán. 25 años de feminismo en el Perú: historia, confluencias y perspectivas (pp. 57-63). Lima: Centro de Estudios Flora Tristán. 
Gutiérrez, B. (2016). Nuevas dinámicas de comunicación, organización y acción social en América Latina. Reconfiguraciones tecnopolíticas. Informe de Investigación de OXFAM. Recuperado de https:/www-cdn.oxfam.org/s3fs-public/file_attachments/ nuevas_dinamicas_de_comunicacion_organizacion_y_accion_social_en_americalatina._reconfiguraciones_tecnopoliticas.pdf.

Natalucci, A. y Rey, J. (2018). ¿Una nueva oleada feminista? Agendas de género, repertorios de acción y colectivos de mujeres (Argentina, 2015-2018). Revista de Estudios Políticos y Estratégicos, 6(2). Recuperado de https://revistaepe.utem.cl/articulos/ una-nueva-oleada-feminista-agendas-de-genero-repertorios-de-accion-y-colectivosde-mujeres-argentina-2015-2018/\#easy-footnote-bottom-13-836.

Puigvert, L. (2001). Las otras mujeres. Barcelona: El Roure.

Rodríguez, P. (2015). \#Ni Una Menos. Vivxs nos queremos. Buenos Aires: Editorial Planeta.

Rovetto, F. (2015). Violencia contra las mujeres: comunicación visual y acción política en "Ni Una Menos" y "Vivas Nos Queremos. Contratexto, 24, 13-34.

Sánchez, A. (2002). El feminismo mexicano antes el movimiento urbano popular. Dos expresiones de lucha de género (1970-1985). Ciudad de México: Editorial Plaza y Valdez.

UNESCO, Consejo Nacional de Educación (2017). Revisión de las políticas educativas 2000-2015. Continuidades en las politicas públicas en educación en Perú: aprendizajes, docentes y gestión descentralizada. Lima: UNESCO.

Vargas, V. (2008). Feminismos en América Latina. Su aporte a la política y a la democracia. Lima: Centro de Estudios Flora Tristán.

Vargas, V. (2004). Los feminismos peruanos: breve balance de tres décadas. En Flora Tristán. 25 años de feminismo en el Perú: historia, confluencias y perspectivas (pp. 15-28). Lima: Centro de Estudios Flora Tristán.

Vargas, V. (1998). Los nudos de la región. En Olea, C. (Ed.). Encuentros, (des)encuentros y búsquedas: el movimiento feminista en América Latina. Lima: Centro de Estudios Flora Tristán.

Vargas, V. (1992). Como cambiar el mundo sin perdernos. El movimiento de mujeres en el Perú y América Latina. Lima: Centro de Estudios Flora Tristán.

Vargas, V. (1989). El aporte de la rebeldía de las mujeres, Lima: Centro de Estudios Flora Tristán.

Villavicencio, M. (1992). Del silencio a la palabra: mujeres peruanas en los siglos XIX y $X X$ [separata]. Lima: Centro de Estudios Flora Tristán. 